\title{
Molecular gas at intermediate redshifts
}

\author{
N. Kanekar and J. N. Chengalur* \\ National Centre for Radio Astrophysics, Post Bag 3, Ganeshkhind, Pune 411 007, India \\ Received 8 November 2001 / Accepted 28 November 2001

\begin{abstract}
We present Giant Metrewave Radio Telescope (GMRT) observations of OH absorption in B3 1504+377 $(z \sim 0.673)$ and PKS $1413+135(z \sim 0.247)$. OH has now been detected in absorption towards four intermediate redshift systems, viz. the lensing galaxies towards B 0218+357 $(z \sim 0.685$; Kanekar et al. 2001) and 1830-211 $(z \sim 0.886$; Chengalur et al. 1999), in addition to the two systems listed above. All four systems also give rise to well studied millimetre wavelength molecular line absorption from a host of molecules, including $\mathrm{HCO}^{+}$. Comparing our $\mathrm{OH}$ data with these millimetre line transitions, we find that the linear correlation between $N_{\mathrm{OH}}$ and $N_{\mathrm{HCO}^{+}}$ found in molecular clouds in the Milky Way (Liszt \& Lucas 1996) persists out to $z \sim 1$. It has been suggested (Liszt \& Lucas 1999) that $\mathrm{OH}$ is a good tracer of $\mathrm{H}_{2}$, with $N_{\mathrm{H}_{2}} / N_{\mathrm{OH}} \approx 10^{7}$ under a variety of physical conditions. We use this relationship to estimate $N_{\mathrm{H}_{2}}$ in these absorbers. The estimated $N_{\mathrm{H}_{2}}$ is $\gtrsim 10^{22}$ in all four cases and substantially different from estimates based on $\mathrm{CO}$ observations.
\end{abstract}

Key words. galaxies: evolution: - galaxies: formation: - galaxies: ISM - cosmology: observations radio lines: galaxies

\section{Introduction}

Molecular hydrogen $\left(\mathrm{H}_{2}\right)$ is the primary constituent of the molecular component of the interstellar medium and plays a crucial role in determining the evolution of the ISM as well as the star formation rate in galaxies. For example, in the Milky Way, $M_{\mathrm{H}_{2}} \sim 5 \times 10^{9} M_{\odot}$, comparable to the mass of the atomic component. Since it is difficult to directly detect $\mathrm{H}_{2}$, its column density, $N_{\mathrm{H}_{2}}$, is usually inferred from observations of other species; these are referred to as tracers of $\mathrm{H}_{2}$ (see e.g. Combes 1999 for a review). The most commonly used tracer of $\mathrm{H}_{2}$ is $\mathrm{CO}$, which is the second most abundant molecule in the ISM. Unfortunately, despite the widespread use of $\mathrm{CO}$ as a tracer of $\mathrm{H}_{2}$, deducing $N_{\mathrm{H}_{2}}$ from $\mathrm{CO}$ observations remains a fairly tricky exercise (see e.g. Liszt \& Lucas 1998, for a discussion).

The $\mathrm{OH}$ column density is known to correlate with the visual extinction $A_{V}$ and, hence, with the total hydrogen column density, $N_{\mathrm{H}}$ (Crutcher 1979). Lucas \& Liszt (1996) and Liszt \& Lucas $(1998,1999)$ examined the variation of $\mathrm{OH}$ and other species (including $\mathrm{H}_{2} \mathrm{CO}$, $\mathrm{HCN}, \mathrm{HNC}$ and $\mathrm{C}_{2} \mathrm{H}$ ) with $\mathrm{HCO}^{+}$and found that most molecules (except $\mathrm{OH}$ ) showed a non-linear dependence on $N_{\mathrm{HCO}^{+}}$, with a rapid increase in their abundances at $N_{\mathrm{HCO}^{+}} \approx 10^{12} \mathrm{~cm}^{-2}$. However, $N_{\mathrm{OH}}$ and $N_{\mathrm{HCO}^{+}}$were found to have a linear relationship extending over more

Send offprint requests to: N. Kanekar,

e-mail: nissim@ncra.tifr.res.in

* e-mail: chengalu@ncra.tifr.res.in than two orders of magnitude in $N_{\mathrm{HCO}^{+}}$(Liszt \& Lucas 1996), with

$$
\frac{N_{\mathrm{HCO}+}}{N_{\mathrm{OH}}} \approx 0.03 \text {. }
$$

Further, the relative abundances of $\mathrm{OH}$ and $\mathrm{HCO}^{+}$to $\mathrm{H}_{2}$ and each other were found to be constant in a variety of galactic clouds (Liszt \& Lucas 1999), with $N_{\mathrm{OH}} / N_{\mathrm{H}_{2}} \approx$ $1 \times 10^{-7}$. Based on these observations, Liszt \& Lucas (1999) suggested that $\mathrm{OH}$ and $\mathrm{HCO}^{+}$were good tracers of $\mathrm{H}_{2}$.

There are presently four known molecular absorption line systems at intermediate redshifts $(z \sim 0.25-0.9)$ with detected $\mathrm{HCO}^{+}$(Wiklind \& Combes 1995, 1996a, 1996b, 1997). Until recently, OH absorption had been detected in only one of these objects, the $z \sim 0.886 \mathrm{ab}-$ sorber towards PKS 1830-211 (Chengalur et al. 1999). We have now carried out a deep search for redshifted $\mathrm{OH}$ absorption in the remaining three absorbers with the GMRT, resulting in detections of absorption in all cases. In this letter, we describe our GMRT observations of two of these absorbers, viz. PKS $1413+135(z=0.2467)$ and B3 $1504+377(z=0.6734)$; the $\mathrm{OH}$ obervations of B $0218+357$ are discussed in Kanekar et al. (2001). We also compare the $\mathrm{OH}$ column densities obtained in the four absorbers with their $\mathrm{HCO}^{+}$column densities and find that the linear relationship between $\mathrm{OH}$ and $\mathrm{HCO}^{+}$ found in the Milky Way persists out to moderate redshifts. Finally, we use the conversion factor suggested by 
Liszt \& Lucas (1999) to estimate $N_{\mathrm{H}_{2}}$ in all these absorbers. Throughout this paper, we use $H_{0}=$ $75 \mathrm{~km} \mathrm{~s}^{-1} \mathrm{Mpc}^{-1}$ and $q_{0}=0.5$.

\section{Observations and data analysis}

The GMRT observations of PKS $1413+135$ and B3 $1504+377$ were carried out in June and October 2001, using the standard 30-station FX correlator. This provides a fixed number of 128 spectral channels over a bandwidth which can be varied between $64 \mathrm{kHz}$ and $16 \mathrm{MHz}$. We used a $4 \mathrm{MHz}$ bandwidth for B3 $1504+377$, thus including both the 1665 and $1667 \mathrm{MHz} \mathrm{OH}$ transitions in the same band and yielding a resolution is $\sim 9.4 \mathrm{~km} \mathrm{~s}^{-1}$. However, in the case of PKS $1413+135$, the $\mathrm{HCO}^{+}$and other millimetre lines have very narrow widths. We hence used a bandwidth of $1 \mathrm{MHz}$ and only observed the $1667 \mathrm{MHz}$ transition (the stronger of the two lines, in thermal equilibrium), with a resolution of $\sim 1.75 \mathrm{~km} \mathrm{~s}^{-1}$. The standard amplitude calibrators 3C 48, 3C 286 and 3C 295 were used for both absolute flux and system bandpass calibration in both cases. No phase calibration was necessary as both PKS 1413+135 and B3 1504+377 are unresolved on even the longest baselines of the GMRT. Only thirteen and seventeen antennas were used for the final spectra of B3 $1504+377$ and PKS $1413+135$, respectively, due to various maintenance activities and debugging; the total on-source times were 6 hours and 5.5 hours respectively.

The data were analysed in AIPS using standard procedures. Continuum emission was subtracted using the AIPS task UVLIN; spectra were then extracted in both cases by simply averaging the source visibilities together, using the AIPS task POSSM, since, as mentioned above, both sources are phase calibrators for the GMRT. Finally, the fluxes of B3 1504+377 and PKS 1413+135 were measured to be $1.2 \mathrm{Jy}$ and $1.6 \mathrm{Jy}$ respectively; our experience with the GMRT indicates that the flux calibration is reliable to $\sim 15 \%$, in this observing mode.

The final GMRT $4 \mathrm{MHz}$ spectrum towards B3 $1504+377$ is shown in Fig. 1a. No smoothing has been applied; the RMS noise is $1.3 \mathrm{mJy}$ per $9.4 \mathrm{~km} \mathrm{~s}^{-1}$ channel. Two absorption lines can be clearly seen in the spectrum, centred at heliocentric frequencies of $995.208 \mathrm{MHz}$ and 996.365 MHz. These correspond to the $1665.403 \mathrm{MHz}$ and $1667.359 \mathrm{MHz}$ transitions of $\mathrm{OH}$, with redshifts $z_{1665}=0.67342 \pm 0.00003$ and $z_{1667}=0.67344 \pm 0.00003$. Note that the redshifts of the lines agree, within our error bars; we will use $z=0.67343$, the average of the redshifts of the two lines, as the redshift of the $\mathrm{OH}$ absorption (but see also the discussion below). The peak line depths are $9.1 \mathrm{mJy}$ and $10.0 \mathrm{mJy}$, implying peak optical depths of $\sim 0.76 \%$ and $0.83 \%$ for the 1665 and $1667 \mathrm{MHz}$ transitions respectively.

The final GMRT $1 \mathrm{MHz}$ spectrum towards PKS $1413+135$ is shown in Fig. 1b. The spectrum has been Hanning smoothed and has an rms noise of $1.3 \mathrm{mJy}$ per $3.5 \mathrm{~km} \mathrm{~s}^{-1}$ channel. Absorption can again be clearly seen, with a peak line flux of $7.9 \mathrm{mJy}$ at a heliocentric frequency of $1337.404 \mathrm{MHz}$. This can be identified with the $1667.359 \mathrm{MHz} \mathrm{OH}$ line, with a redshift $z=0.24671 \pm 0.00001$. The peak optical depth is $0.49 \%$.

\section{Discussion}

For an optically thin cloud in thermal equilibrium, the $\mathrm{OH}$ column density of the absorbing gas $N_{\mathrm{OH}}$ is related to the excitation temperature $T_{x}$ and the $1667 \mathrm{MHz}$ optical depth $\tau_{1667}$ by the expression (e.g. Liszt \& Lucas 1996)

$N_{\mathrm{OH}}=2.24 \times 10^{14}\left(\frac{T_{x}}{f}\right) \int \tau_{1667} \mathrm{~d} V$

where $f$ is the covering factor of the absorber. In the above, $N_{\mathrm{OH}}$ is in $\mathrm{cm}^{-2}, T_{x}$ in $\mathrm{K}$ and $\mathrm{d} V$ in $\mathrm{km} \mathrm{s}^{-1}$. VLBI observations, when available, can be used to constrain the extent of the background radio continuum, and hence to estimate the covering factor $f$. Unfortunately, the $\mathrm{OH}$ excitation temperature $T_{x}$ cannot be directly estimated, for cosmologically distant objects. In the Galaxy, OH emission studies have shown that this temperature may be as low as $T_{x} \sim T_{\mathrm{CMB}}+1 \mathrm{~K}$, with similar values for the $\mathrm{HCO}^{+}$ line $\left(T_{x}\left(\mathrm{HCO}^{+}\right) \sim T_{\mathrm{CMB}}\right.$; Lucas \& Liszt 1996). However, the excitation temperatures of the redshifted $\mathrm{HCO}^{+}$lines in three of the four absorbers have been found to be higher than $T_{\mathrm{CMB}}(1+z)$, the redshifted CMB temperature; it is thus quite likely that the $\mathrm{OH}$ excitation temperature too will be higher in these systems. Given that all four absorption systems are believed to originate either in spiral disks or in late-type galaxies (Lehár et al. 2000; Stocke et al. 1992; Stickel \& Kühr 1994), we will, in the absence of additional information, assume $T_{x}=10 \mathrm{~K}$, a typical temperature in dark clouds in the Milky Way.

B3 1504+377: The OH absorption redshift, $z=0.67343$, is in good agreement with that of the HI $(z=0.67340$; Carilli et al. 1997); however, the molecular absorption seen in the millimetre wave bands peaks about $15 \mathrm{~km} \mathrm{~s}^{-1}$ away, at $z=0.67335$ (Wiklind \& Combes 1996a). Next, while the two $z=0.67343 \mathrm{OH}$ lines are not very different in peak optical depth, the $1667 \mathrm{MHz}$ line can be seen to be the wider of the two (total spread $\sim 103 \mathrm{~km} \mathrm{~s}^{-1}$ against $\sim 75 \mathrm{~km} \mathrm{~s}^{-1}$ ); these widths are similar to those of the $z=0.67335 \mathrm{~mm}$-wave lines (total spread $\sim 100 \mathrm{~km} \mathrm{~s}^{-1}$; Wiklind \& Combes 1996a). The integrated optical depths of the $\mathrm{OH}$ lines are $\int \tau_{1665} \mathrm{~d} V=0.257 \mathrm{~km} \mathrm{~s}^{-1}$ and $\int \tau_{1667} \mathrm{~d} V=0.448 \mathrm{~km} \mathrm{~s}^{-1}$. We note that millimetre wave molecular absorption has also been detected at $z=0.67150$ (Wiklind \& Combes 1996a); the $1665 \mathrm{MHz}$ $\mathrm{OH}$ line corresponding to this redshift arises at a heliocentric frequency of $996.352 \mathrm{MHz}$, and may thus overlap with the $1667 \mathrm{MHz}$ line of the $z=0.67343 \mathrm{ab}-$ sorber. We will hence use the integrated optical depth in the $1665 \mathrm{MHz}$ line of the $z=0.67343$ absorber to evaluate its $\mathrm{OH}$ column density (assuming thermal equilibrium, i.e. $\left.\int \tau_{1667} \mathrm{~d} V / \int \tau_{1665} \mathrm{~d} V=1.8\right)$. The integrated $1665 \mathrm{MHz}$ optical depth then yields an $\mathrm{OH}$ column density $N_{\mathrm{OH}}=1.04 \times\left(T_{x} / f\right) \times 10^{14} \mathrm{~cm}^{-2}$. Carilli et al. (1997) estimate $f \geq 0.46$, from VLBI observations at 

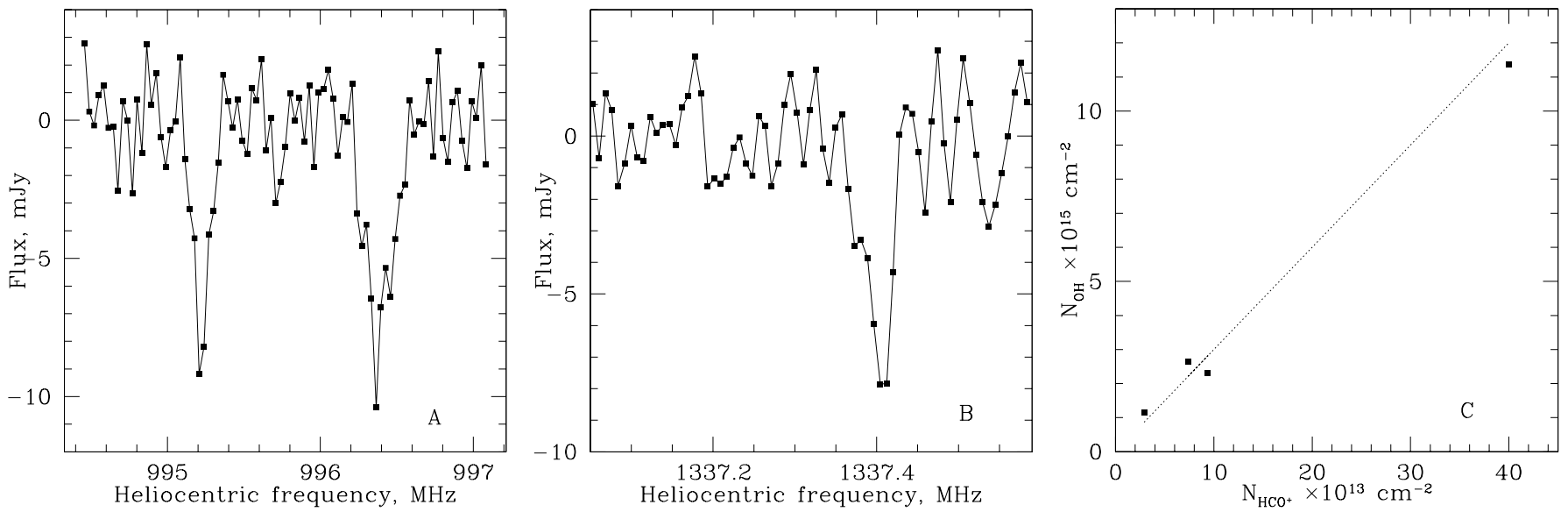

Fig. 1. a) $9.4 \mathrm{~km} \mathrm{~s}^{-1}$ resolution $\mathrm{OH}$ spectrum towards B3 $1504+377$. The spectrum includes the 1665 \& $1667 \mathrm{OH}$ lines. b) $3.5 \mathrm{~km} \mathrm{~s}^{-1}$ resolution spectrum of $1667 \mathrm{OH}$ line towards PKS $1413+135$. c) $N_{\mathrm{OH}}$ versus $N_{\mathrm{HCO}}+$ for the four absorbers of our sample. The dotted line shows the correlation found in molecular clouds in the Milky Way, and is not a fit. Note that the uncertainty in $N_{\mathrm{OH}}$ is dominated by the uncertainties in $T_{x}$ and the covering factor $f$; the statistical errors on the optical depth are small $(<10 \%)$ in all cases.

Table 1. Summary of $\mathrm{OH}$ absorption studies.

\begin{tabular}{|c|c|c|c|c|c|c|c|}
\hline Source & $z_{\text {abs }}$ & $\begin{array}{c}N_{\mathrm{OH}} \\
10^{15} \mathrm{~cm}^{-2}\end{array}$ & $\begin{array}{c}N_{\mathrm{HCO}^{+}}{ }^{\star} \\
10^{13} \mathrm{~cm}^{-2}\end{array}$ & $\begin{array}{c}N_{\mathrm{HCO}+}{ }^{\dagger} \\
(\text { Obs.) } \\
10^{13} \mathrm{~cm}^{-2}\end{array}$ & $\begin{array}{c}N_{\mathrm{H}_{2}}^{\ddagger} \\
(\mathrm{OH}) \\
10^{22} \mathrm{~cm}^{-2}\end{array}$ & $\begin{array}{c}N_{\mathrm{H}_{2}}{ }^{*} \\
(\mathrm{CO}) \\
10^{22} \mathrm{~cm}^{-2}\end{array}$ & $A_{V}$ \\
\hline PKS 1413+135 & 0.24671 & 1.16 & 3.5 & 2.9 & 1.16 & 0.04 & 17.8 \\
\hline B3 $1504+377$ & 0.67343 & 2.3 & 6.9 & 9.4 & 2.3 & 0.12 & 27.1 \\
\hline B $0218+357$ & 0.68468 & 2.65 & 7.8 & 7.4 & 2.65 & 40.0 & 28.9 \\
\hline PKS 1830-211 & 0.88582 & 11.38 & 34.2 & 40 & 11.38 & 4.0 & 123.2 \\
\hline
\end{tabular}

* Obtained using Eq. (1).

$\dagger$ Actual measured $\mathrm{HCO}^{+}$column densities, from Wiklind \& Combes (1995), (1996a), (1997), (1998) and Menten et al. (1999).

$\ddagger$ Obtained using $N_{\mathrm{H}_{2}}=1.0 \times 10^{7} \times N_{\mathrm{OH}}$.

* Estimated from CO observations, from Wiklind \& Combes (1996a), (1996b), (1997), (1999).

1.6 and $5 \mathrm{GHz}$, with the lower value obtained if only the compact core of the radio continuum (size $\approx 7.2 \mathrm{pc}$ ) is covered by the absorbing cloud. On the other hand, $f=0.74$, if the radio jet of the source is also covered; this would require a cloud of size greater than $\sim 54$ pc. Typical sizes of Giant Molecular Clouds in the Milky Way range from 10 to $50 \mathrm{pc}$ (Blitz 1990); we will hence use a covering factor $f=0.46$ in the analysis. This yields $N_{\mathrm{OH}}=2.3 \times 10^{15}\left(T_{x} / 10\right)(0.46 / f) \mathrm{cm}^{-2}$.

PKS 1413+135: The redshift of the $\mathrm{OH}$ absorption towards PKS 1413+135 is in excellent agreement with that of the millimetric absorption $(z=0.24671$; Wiklind \& Combes 1997). The width of the $1667 \mathrm{MHz}$ line is also quite narrow, with a total spread $\sim 14 \mathrm{~km} \mathrm{~s}^{-1}$ (slightly wider than the mm lines of Wiklind \& Combes (1997), which have a total spread $\lesssim 10 \mathrm{~km} \mathrm{~s}^{-1}$ ); the integrated optical depth is $\tau_{1667}=0.023 \pm 0.001 \mathrm{~km} \mathrm{~s}^{-1}$. Equation (2) then yields an $\mathrm{OH}$ column density $N_{\mathrm{OH}}=0.51 \times\left(T_{x} / f\right) \times$ $10^{14} \mathrm{~cm}^{-2}$. VLBA maps of PKS $1413+135$ at $3.6,6,13$ and $18 \mathrm{~cm}$ (Perlman et al. 1996) have shown that the core (component $\mathrm{N}$ in their maps) is highly inverted, with a spectral index $\alpha=+1.7$. Extrapolating their flux measurements yields a core flux of $\sim 70$ mJy at the GMRT observing frequency, within $\sim 3$ mas (i.e. $\sim 10 \mathrm{pc}$ at $z=0.247$ ). Since this is likely to be covered by the molecular cloud, we obtain a lower limit on the covering factor, $f \geq 0.044$. On the other hand, if components C and D (Perlman et al. 1996) are also covered, it would imply $f \sim 0.2$ (using extrapolated fluxes of components $\mathrm{C}$ and $\mathrm{D}$ ). This is, however, unlikely, given that components $\mathrm{C}, \mathrm{D}$ and the core are spread over $\sim 20$ mas (i.e. $\sim 65 \mathrm{pc}$ at $z=0.247$ ), larger than the size of a typical molecular cloud. We will use $f=0.044$ in the analysis (note that $f \sim 0.1$ is also possible); this yields $N_{\mathrm{OH}}=1.16 \times 10^{15}\left(T_{x} / 10\right)(0.044 / f) \mathrm{cm}^{-2}$.

We also evaluate $N_{\mathrm{OH}}$ for the other two high redshift molecular absorbers in which $\mathrm{OH}$ absorption has been detected, towards B 0218+357 and PKS 1830-211 (Chengalur et al. 1999; Kanekar et al. 2001). In the case of PKS 1830-211, the integrated optical depth in the $1667 \mathrm{MHz}$ line is $\int \tau_{1667} \mathrm{~d} V=1.83 \mathrm{~km} \mathrm{~s}^{-1}$; i.e. $N_{\mathrm{OH}}=4.1 \times 10^{14}\left(T_{x} / f\right) \mathrm{cm}^{-2}$. The millimetric absorption is known to occur only towards the south-west component of the background source, which contains $\sim 36 \%$ of the 
radio flux (Wiklind \& Combes 1998); the covering factor is thus likely to be $f \sim 0.36$. We then obtain (again using $\left.T_{x}=10 \mathrm{~K}\right) N_{\mathrm{OH}}=11.4 \times 10^{15}\left(T_{x} / 10\right)(0.36 / f) \mathrm{cm}^{-2}$. Similarly, the integrated optical depth in the $1667 \mathrm{MHz}$ line is $\int \tau_{1667} \mathrm{~d} V=0.772 \mathrm{~km} \mathrm{~s}^{-1}$, in the case of the $z=0.6846$ absorber towards B $0218+357$ (Kanekar et al. $2001)$; thus, $N_{\mathrm{OH}}=1.1 \times 10^{14} \times\left(T_{x} / f\right) \mathrm{cm}^{-2}$. Carilli et al. (1993) estimate the covering factor to be $f \sim 0.4$, assuming that only component A of the background continuum source is covered by the absorbing cloud. The latter is reasonable since it is known that the millimetric absorption also only occurs against this component (Wiklind \& Combes 1995). The OH column density is then $N_{\mathrm{OH}}=2.65 \times 10^{15}\left(T_{x} / 10\right)(0.4 / f) \mathrm{cm}^{-2}$.

Figure 1c shows a plot of the $\mathrm{OH}$ column density versus the $\mathrm{HCO}^{+}$column density for the four absorbers of our sample. The dotted line is the relationship found in the Milky Way. All four systems lie close to this line; the linear relationship between $\mathrm{OH}$ and $\mathrm{HCO}^{+}$clearly appears to persist out to moderate redshifts. Table 1 summarises our results and also lists the $\mathrm{H}_{2}$ column densities (evaluated using $N_{\mathrm{OH}} / N_{\mathrm{H}_{2}}=10^{-7}$ ) for the four absorbers of our sample; these can be seen to be quite different from the values estimated from $\mathrm{CO}$ observations (penultimate column). It is interesting that our estimate of the $\mathrm{H}_{2}$ column density in the $z=0.6846$ absorber towards B $0218+357$ is in reasonable agreement with that obtained by Gerin et al. (1997) $\left(N_{\mathrm{H}_{2}}=2 \times 10^{22} \mathrm{~cm}^{-2}\right)$, using the ${ }^{17} \mathrm{CO}$ line. We also note that the good agreement between the observed $\mathrm{HCO}^{+}$column densities and those estimated using Eq. (1) is despite the fact that we have used the general excitation temperature, $T_{x}=10 \mathrm{~K}$, for the $\mathrm{OH}$ line in all cases. For example, the $\mathrm{HCO}^{+}$excitation temperature is measured to be $13 \mathrm{~K}$, in the case of the $z=0.6734 \mathrm{ab}-$ sorber towards B3 $1504+377$; if this value were also used for $T_{x}$, one would obtain $N_{\mathrm{HCO}^{+}}=9.0 \times 10^{13} \mathrm{~cm}^{-2}$, in even better agreement with that obtained from the $\mathrm{HCO}^{+}$ absorption spectra of Wiklind \& Combes (1996a).

Finally, the last column of Table 1 gives the visual extinction along the four lines of sight, evaluated using the $\mathrm{H}_{2}$ column densities of Col. 8, the HI column densities obtained assuming a spin temperature of $100 \mathrm{~K}$ (Carilli et al. 1992; Carilli et al. 1993; Carilli et al. 1997; Chengalur et al. 1999), and a Galactic extinction law $\left(R_{V}=3.1\right.$; Binney \& Merrifield 1998). We note that a far lower extinction $\left(A_{V} \sim 28\right)$ is obtained towards B $0218+357$ than that estimated from observations of the ${ }^{12} \mathrm{CO}$ line ( $A_{V} \gtrsim 500 ;$ Wiklind \& Combes 1999). While $A_{V}=28$ is still quite large and does require the presence of fine structure in the molecular cloud (since component A is visible in the optical; Wiklind \& Combes 1999), the present value requires a less dramatic change in physical conditions across the optical source than that obtained by Wiklind \& Combes (1999). We also find a high extinction towards PKS $1413+135\left(A_{V} \sim 18\right)$, although still somewhat smaller than that obtained from the deficit of soft X-rays $\left(A_{V} \gtrsim 30\right.$; Stocke et al. 1992).
In summary, we find that the linear relationship between $\mathrm{OH}$ and $\mathrm{HCO}^{+}$column densities, seen in Galactic molecular clouds, appears to persist out to absorbers at intermediate redshift, with $N_{\mathrm{HCO}^{+}} \approx 0.03 \times N_{\mathrm{OH}}$. One may thus be able to use $\mathrm{OH}$ absorption lines to trace the $\mathrm{H}_{2}$ content of molecular clouds at cosmological distances; all four absorbers of our sample have $N_{\mathrm{H}_{2}} \gtrsim 10^{22} \mathrm{~cm}^{-2}$.

Acknowledgements. The GMRT observations presented in this paper would not have been possible without the many years of dedicated effort put in by the GMRT staff to build the telescope. The GMRT is run by the National Centre for Radio Astrophysics of the Tata Institute of Fundamental Research. We thank Chris Carilli for illuminating discussions, which were useful in planning the observations.

\section{References}

Blitz, L. 1990, in The Evolution of the Interstellar Medium, ed. L. Blitz, ASP Conf. Ser., 12, 273

Binney, J. J., \& Merrifield, M. 1998, Galactic Astron. (Princeton University Press, Princeton, NJ)

Carilli, C. L., Perlman, E. S., \& Stocke, J. T. 1992, ApJ, 400, L13

Carilli, C. L., Rupen, M. P., \& Yanny, B. 1993, ApJ, 412, L59

Carilli, C. L., Menten, K. M., Reid, M. J., \& Rupen, M. P. 1997, ApJ, 474, L89

Chengalur, J. N., de Bruyn, A. G., \& Narasimha, D. 1999, A\&A, 343, L79

Combes, F. 1999, in $\mathrm{H}_{2}$ in Space, ed. F. Combes, \& G. Pineau des Forêts (Cambridge University Press), Astrophysics Ser., 46

Crutcher, R. M. 1979, ApJ, 239, 881

Gerin, M., Phillips, T. G., Benford, D. J., et al. 1997, ApJ, 488, L31

Kanekar, N., et al. 2001, in preparation

Lehár, J., et al. 2000, ApJ, 536, 584

Liszt, H., \& Lucas, R. 1996, A\&A, 314, 917

Lucas, R., \& Liszt, H. 1996, A\&A, 307, 237

Liszt, H., \& Lucas, R. 1998, A\&A, 339, 561

Liszt, H., \& Lucas, R. 1999, in Highly Redshifted Radio Lines, ed. C. L. Carilli, S. J. E. Radford, K. M. Menter, \& G. I. Langston, ASP Conf. Ser., 156, 188

Menten, K. M., Carilli, C. L., \& Reid, M. J., in Highly Redshifted Radio Lines, ed. C. L. Carilli, S. J. E. Radford, K. M. Menter, \& G. I. Langston, ASP Conf. Ser., 156, 218

Perlman, E. S., Carilli, C. L., Stocke, J. T., \& Conway, J. 1996, AJ, 111, 1839

Stickel, M., \& Kühr, H., A\&AS, 105, 67

Stocke, J. T., Wurtz, R., Wang, Q., Elston, R., \& Jannuzi, B. T. 1992, ApJ, 400, L17

Wiklind, T., \& Combes, F. 1995, A\&A, 299, 382

Wiklind, T., \& Combes, F. 1996a, A\&A, 315, 86

Wiklind, T., \& Combes, F. 1996b, Nature, 379, 139

Wiklind, T., \& Combes, F. 1997, A\&A, 328, 48

Wiklind, T., \& Combes, F. 1998, ApJ, 500, 129

Wiklind, T., \& Combes, F. 1999, in Highly Redshifted Radio Lines, ed. C. L. Carilli, S. J. E. Radford, K. M. Menter, \& G. I. Langston, ASP Conf. Ser., 156, 202 